\title{
TEMPERATURAS DE ACIONAMENTO DE SISTEMAS DE CLIMATIZAÇÃO PARA PERUS EM ÉPOCAS DE BAIXA UMIDADE RELATIVA DO AR
}

\author{
ANGÉLICA S. MENDES ${ }^{1}$, DANIELLA J. DE MOURA², IRENILZA DE A. NÄ̈̈̈ ${ }^{3}$, \\ LÍLIA T. SONODA ${ }^{4}$
}

\begin{abstract}
RESUMO: Este estudo teve o objetivo de avaliar distintos programas de acionamento dos sistemas de climatização (ventiladores e nebulizadores), visando a aprimorar o ambiente interno e o bemestar das aves, evitando possíveis perdas produtivas e gastos excessivos em energia elétrica. O estudo foi conduzido em dois aviários localizados no município de Uberlândia - MG, durante a época de baixa umidade relativa do ar (junho a dezembro). Os aviários foram comparados sob os parâmetros de concentrações de $\mathrm{CO}_{2}$, temperatura e umidade relativa do ar, temperatura superficial da cama e índices produtivos. Os dados climáticos foram submetidos à análise de variância, e os dados produtivos submetidos ao teste de Qui-quadrado, utilizando-se do software MINITAB V.14. Os resultados apontados pelos tratamentos 1 e 2 mostraram que as concentrações de $\mathrm{CO}_{2}$ no ar (372,70 e 379,46 ppm, respectivamente) estiveram abaixo dos limites máximos recomendados internacionalmente para ambos os sistemas. As temperaturas internas $\left(26,23\right.$ e $26,56{ }^{\circ} \mathrm{C}$, respectivamente) estiveram sempre acima do recomendado para as aves, e os níveis de umidade relativa do ar (69,73 e 70,42, respectivamente) estiveram, em média, na faixa recomendada, para ambos os sistemas. Conclui-se que o aviário programado para acionar o sistema de climatização mais tardiamente consumiu menos energia elétrica, apresentou melhor conversão alimentar, menor incidência de artrite, porém maior percentual de mortalidade.
\end{abstract}

PALAVRAS-CHAVE: ventiladores, nebulizadores, conforto térmico, consumo de energia elétrica.

\section{ACTIVATING TEMPERATURES OF VENTILATION SYSTEMS FOR TURKEYS IN LOW HUMIDITY PERIODS}

\begin{abstract}
This study aimed to evaluate different turn on systems of climatization (fans and fogging sprinklers) to improve the internal environment and the birds' welfare, avoiding productive losses and expensive wastes in electrical energy. The study was conducted in a commercial property with two poultry houses, located in Uberlândia, MG, Brazil, during the phase of low relative humidity of the air (June to December). The poultry houses were compared under the parameters of concentration of $\mathrm{CO}_{2}$, temperature and relative humidity, surface temperature of the litter and production indexes. The climatic data were subjected to analysis of variance and productive data submitted to chi-square, using the software Minitab, V.14. The results showed in treatments one and two that concentrations of $\mathrm{CO}_{2}$ in the air $(372,70$ and $379,46 \mathrm{ppm}$, respectively) were below the internationally recommended limits for both systems. The internal temperatures $\left(26,23\right.$ e $26,56{ }^{\circ} \mathrm{C}$, respectively) were always above the recommended for birds, and the levels of relative humidity $(69,73$ e 70,42 , respectively) were on average in the recommended range for both systems. The conclusion is that the poultry house programmed to trigger the air-conditioning later, consumed less electricity, had better feed conversion, lower incidence of arthritis, but a higher percentage of mortality.
\end{abstract}

KEYWORDS: fans, fogging sprinklers, thermal comfort, electric power consumption.

\footnotetext{
${ }^{1}$ Profa. Adjunta, Universidade Tecnológica Federal do Paraná, Dois Vizinhos - PR.

${ }^{2}$ Profa. Doutora, Conselho de Infraestrutura Rural, Faculdade de Engenharia Agrícola, UNICAMP, Campinas - SP.

${ }^{3}$ Profa. Titular, Conselho de Infraestrutura Rural, Faculdade de Engenharia Agrícola, UNICAMP, Campinas - SP.

${ }^{4}$ Mestranda, Conselho de Infraestrutura Rural, Faculdade de Engenharia Agrícola, UNICAMP, Campinas - SP.

Recebido pelo Conselho Editorial em: 28/8/2009

Aprovado pelo Conselho Editorial em: 13/6/2010
} 


\section{INTRODUÇÃO}

O mercado de produtos à base de carne de peru tem-se mostrado bastante promissor não só no Brasil como em vários outros países, representando 6,9\% da produção mundial. Entre 2005 e 2009, houve um incremento de $29,8 \%$ no total produzido. A tecnificação e o controle sanitário foram as principais alavancas de crescimento desse setor, que continua despertando o interesse dos produtores como alternativa para diversificar a produção (UBA, 2008).

Os perus, ao contrário dos frangos de corte, necessitam de duas instalações distintas para completar o ciclo do primeiro dia de vida até o abate: a primeira é chamada de fase inicial de criação, e a segunda, fase final de criação (CAVALCHINI, 1985). Ambas as fases necessitam de um sistema de ventilação adequado, pois o objetivo da ventilação é o fornecimento de ar fresco e o alcance dos requisitos de qualidade de ar e de manutenção do aquecimento/resfriamento para os animais alojados. Logo, o sucesso no fornecimento de um ambiente adequado de alojamento às aves depende, principalmente, do sistema de ventilação utilizado (WANG et al., 1999).

O manejo para as práticas de uma boa qualidade de ar necessita de sistemas de resfriamento e/ou aquecimento e ventilação que promovam um ambiente equilibrado. Uma consequência de um ambiente não equilibrado é a incidência de doenças respiratórias nas aves (ZEJDA et al., 1994; SENTHILSELVAN et al., 1997). Umidade relativa e temperatura do ar também possuem impacto na qualidade do ar, por influenciar na proliferação e sobrevivência de alguns patógenos e de severas doenças (CARVALHO, 1991) e no bem-estar e desempenho das aves (YALÇIN et al., 1997; WATHES et al., 1998).

Neste contexto, o controle ambiental adequado para os aviários torna-se um fator imprescindível para a melhoria da produtividade. No entanto, a complexidade do controle do sistema de climatização, ou seja, a programação ideal de acionamento destes sistemas a ser adotada em função da temperatura, considerando concomitantemente a economia em energia elétrica e o investimento inicial, dificulta o alcance de um adequado ambiente para as aves. Isto indica que grande esforço deve ser realizado para identificar as falhas em aclimatação e, em seguida, melhorar a eficiência da ventilação. No entanto, sabe-se que há pouca informação na literatura sobre o uso de dados do ambiente de alojamento no dimensionamento e programação dos sistemas de climatização (LEE et al., 2003).

Considerando que a produção intensiva de perus pode ser aprimorada, gerando fonte de receita para novos investidores, faz-se necessário o estudo do ambiente interno dos aviários onde estas aves estarão ou já estão inseridas e da maximização dos equipamentos de climatização responsáveis pela refrigeração ambiental. Desta forma, este trabalho foi conduzido com o objetivo de avaliar distintos programas de acionamento dos sistemas de climatização (ventiladores e nebulizadores), visando a aprimorar o ambiente interno e o bem-estar das aves, evitando possíveis perdas produtivas e gastos excessivos em energia elétrica.

\section{MATERIAL E MÉTODOS}

Este estudo foi realizado em uma propriedade com dois aviários comerciais de perus na fase final de criação, situada no município de Uberlândia - MG. Os aviários possuíam as mesmas dimensões $(125,00 \times 12,00 \mathrm{~m})$, pé-direito de $3 \mathrm{~m}$, orientação ( $\mathrm{L}$ - O) e idêntico aporte de equipamentos. O sistema de nebulização para ambos os aviários era composto por um motor de $2 \mathrm{cv}$ e bicos de média pressão, espaçados a cada $3 \mathrm{~m}$ e distribuídos em linhas longitudinais. O sistema de ventilação era por pressão positiva, com 16 ventiladores de $0,5 \mathrm{cv}$ em cada aviário, distribuídos em linhas longitudinais e divididos em dois grupos de acionamento: o primeiro grupo acionava oito ventiladores, e o segundo grupo acionava os oito ventiladores restantes.

O funcionamento dos controladores dava-se basicamente por meio de termostatos. Em cada aviário, havia dois sensores de temperatura conectados à central de controle dos ventiladores, localizados nas áreas inicial e final dos aviários. A média dos dois sensores era informada à central 
de controle. Entretanto, os aviários (tratamentos) diferenciavam-se pelas temperaturas de acionamento dos sistemas de climatização, conforme se observa na Tabela 1.

TABELA 1. Temperaturas de acionamento dos sistemas de climatização $($ vent $=$ ventiladores; neb $=$ nebulizadores), para cada faixa etária das aves e tratamentos. Turn on temperature of climatization systems (vent $=$ fans; neb =fog sprinklers) for each age group of birds and treatments.

\begin{tabular}{|c|c|c|c|}
\hline $\begin{array}{l}\text { Tratamento/ } \\
\text { Idade }\end{array}$ & I1: 28-60 dias & I2: $61-100$ dias & I3: $101-150$ dias \\
\hline A1 & $\begin{array}{l}30^{\circ} \mathrm{C}\left(1^{\mathrm{o}} \text { grupo vent }\right) \\
31^{\circ} \mathrm{C} \text { (grupo neb); } \\
32^{\circ} \mathrm{C}\left(2^{\mathrm{o}} \text { grupo vent }\right) .\end{array}$ & $\begin{array}{l}29^{\circ} \mathrm{C}\left(1^{\mathrm{o}} \text { grupo vent }\right) \\
30^{\circ} \mathrm{C} \text { (grupo neb); } \\
31^{\circ} \mathrm{C}\left(2^{\circ} \text { grupo vent }\right)\end{array}$ & $\begin{array}{l}28^{\circ} \mathrm{C}\left(1^{\mathrm{o}} \text { grupo vent }\right) ; \\
29^{\circ} \mathrm{C} \text { (grupo neb); } \\
30^{\circ} \mathrm{C}\left(2^{\mathrm{o}} \text { grupo vent }\right) .\end{array}$ \\
\hline A 2 & $\begin{array}{l}26^{\circ} \mathrm{C}\left(1^{\mathrm{o}} \text { grupo vent }\right) \\
27^{\circ} \mathrm{C} \text { (grupo neb); } \\
28^{\circ} \mathrm{C}\left(2^{\mathrm{o}} \text { grupo vent }\right) .\end{array}$ & $\begin{array}{l}25^{\circ} \mathrm{C}\left(1^{\mathrm{o}} \text { grupo vent }\right) \\
26^{\circ} \mathrm{C} \text { (grupo neb); } \\
\left.27^{\circ} \mathrm{C} \text { ( } 2^{\mathrm{o}} \text { grupo vent }\right)\end{array}$ & $\begin{array}{l}24^{\circ} \mathrm{C}\left(1^{\mathrm{o}} \text { grupo vent }\right) ; \\
25^{\circ} \mathrm{C} \text { (grupo neb); } \\
26^{\circ} \mathrm{C}\left(2^{\mathrm{o}} \text { grupo vent }\right) .\end{array}$ \\
\hline
\end{tabular}

O período do estudo foi durante a época de baixa umidade relativa do ar, que ocorreu entre os meses de junho e dezembro de 2007 (UR média de 57\%), conforme análise do banco de dados meteorológicos (temperatura do ar, UR do ar, precipitação e cobertura do céu) do Instituto de Geografia da Universidade Federal de Uberlândia, dos últimos dez anos. Em cada aviário, foram alojadas um total de 4.500 aves de mesma origem e sexo (machos), com idade inicial de 28 dias e permaneceram no experimento até a idade final para abate (150 dias).

Cada aviário foi dividido virtualmente em 24 células para o registro da concentração de dióxido de carbono $\left(\mathrm{CO}_{2}\right)$, temperatura ambiente $(\mathrm{T})$, umidade relativa (UR) e temperatura superficial da cama (TC), na altura das aves. Foram utilizados 15 segundos para o monitoramento climático em cada uma das 24 células avaliadas e mais 10 segundos para a estabilização dos equipamentos. O registro dos dados foi realizado sistematicamente na direção oeste-leste do galpão, durante os períodos: diurno ( $8 \mathrm{~h}$ ), o mais crítico do dia em termos de calor $(14 \mathrm{~h})$, e o período do entardecer (17 h), segundo metodologia proposta por MIRAGLIOTTA (2005).

Para os registros de T e UR, utilizou-se um equipamento da marca Instrutherm ${ }^{\circledR}$, modelo THAL-300. Para TC, utilizou-se um termômetro infravermelho digital da marca Instrutherm ${ }^{\circledR}$, modelo TI-900, e para a coleta das concentrações de $\mathrm{CO}_{2}$, um Indoor Air Quality Meter - modelo 8732 (IAQ-CALCTM).

Mensuraram-se as seguintes variáveis produtivas: peso médio semanal, ganho de peso vivo das aves, conversão alimentar, mortalidade e incidência de enfermidades: aerossaculite, dermatose e artrite. A pesagem semanal das aves foi realizada por meio de amostragem: capturavam-se 80 aves aleatoriamente no início, meio e fím do aviário, e com auxílio de uma balança de pendura determinava-se o peso médio das aves. A incidência de enfermidades foi mensurada no abatedouro. Além disso, avaliou-se o consumo de energia elétrica por meio de medidores instalados em cada aviário.

Posteriormente, os dados climáticos foram submetidos à Análise de Variância, e os dados produtivos, ao teste do Qui-quadrado, utilizando-se do software MINITAB V.14.

\section{RESULTADOS E DISCUSSÃO}

Conforme o resultado das Análises de Variância, comprovou-se que não houve diferença significativa entre os tratamentos na distribuição das seguintes variáveis climáticas: concentrações de $\mathrm{CO}_{2}(\mathrm{P}=0,065)$, umidade relativa $(\mathrm{P}=0,108)$ e temperatura superficial da cama $(\mathrm{P}=0,071)$. Porém, comprovou-se diferença significativa para a variável temperatura ambiente $(\mathrm{P}=0,001)$. 
Por meio da Figura 1a, observa-se que o A1 apresentou a maior média de temperatura ambiente, porém menor média de UR (Tabela 2). Ambos os resultados eram esperados, pois o tratamento A1 acionava o sistema de climatização tardiamente em comparação com o A2 e a UR, conforme as próprias características psicrométricas do ar se reduzem frente às temperaturas mais elevadas (MIRAGLIOTTA, 2005). Porém, os resultados médios estiveram dentro da faixa de conforto térmico recomendada para as aves pela ASHRAE (2002), entre 60 a 70\%, para ambos os tratamentos.
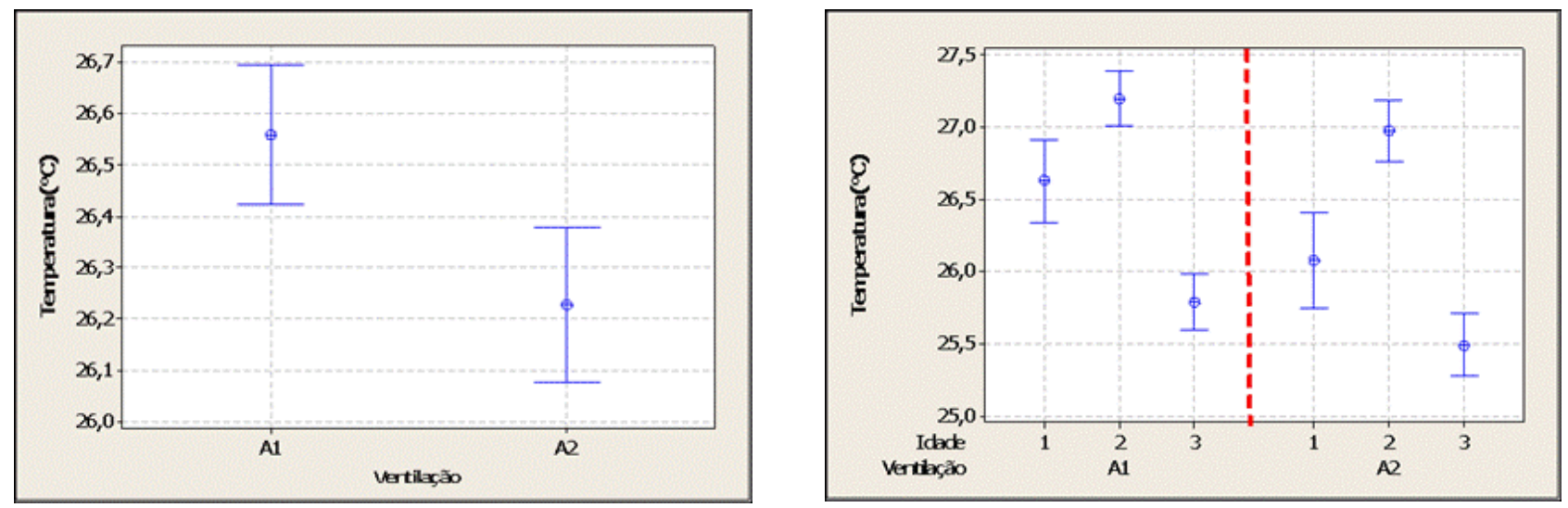

FIGURA 1. Temperaturas médias em função dos tratamentos (a) e da idade do lote (b). Average temperatures in function of treatments (a) and flock age (b).

Observa-se, na Figura 1b, que a temperatura apresentou variação similar entre os tratamentos ao longo do crescimento do lote. Ambos os tratamentos apresentaram menores níveis de T para a idade mais avançada do lote. Explica-se esse fato pelo acionamento dos sistemas de climatização, pois foram programados em função das exigências térmicas das aves, ou seja, aves mais velhas necessitam de menores temperaturas ambiente. Entretanto, observa-se que ambos os tratamentos forneceram um ambiente com temperaturas superiores ao desejado pelas aves, conforme recomendações técnicas (MANUAL DE BOAS PRÁTICAS DA NICHOLAS, 2007): conforme idade $1\left(21^{\circ} \mathrm{C}\right)$, idade $2\left(16^{\circ} \mathrm{C}\right)$ e idade $3\left(14^{\circ} \mathrm{C}\right)$ (VAN DER HEL et al., 1992). O ambiente com temperaturas elevadas conduz ao decréscimo no desempenho das aves e piora na conversão alimentar, o que justifica o gasto em energia elétrica pelo uso adequado dos sistemas de climatização (TABELA 2).

XIN et al. (1998) relataram, em estudo com perus machos, que os níveis de umidade relativa do ar e de temperatura ambiente se modificam em função do sistema de ventilação utilizado. Outros estudos constatam a dificuldade de manter um ambiente adequado e uniforme em grandes instalações para frangos de corte (SEO et al., 2006). Para ANDONOV et al. (2003) \& MUTAF et al. (2004), existe conexão entre taxas eficazes de ventilação e o design do sistema, comprovada em experimentos com suínos e frangos de corte. WANG \& ZHANG (1999) também relataram que a temperatura e a velocidade do ar estão interligadas ao modelo de sistema de ventilação adotado. Entretanto, outros autores relatam que a eficácia da ventilação é afetada primeiramente pelo sistema de ventilação e, posteriormente, pela programação da taxa de ventilação (ZHANG et al., 2001).

TABELA 2. Valores médios para as variáveis avaliadas frente aos tratamentos. Average values for the evaluated variables on different treatments.

\begin{tabular}{lcc}
\hline Variável/Tratamento & $\mathrm{A} 1$ & $\mathrm{~A} 2$ \\
\hline Temperatura $\left({ }^{\circ} \mathrm{C}\right)$ & 26,56 & 26,23 \\
$\mathrm{UR}(\%)$ & 69,73 & 70,42 \\
$\mathrm{CO}_{2}(\mathrm{ppm})$ & 372,70 & 379,46 \\
$\mathrm{TC}\left({ }^{\circ} \mathrm{C}\right)$ & 27,35 & 28,12 \\
\hline
\end{tabular}


Independentemente do programa de acionamento utilizado, as médias de concentração de $\mathrm{CO}_{2}$ mantiveram-se em níveis bem inferiores ao limite permitido para a criação de perus, pois a concentração máxima de $\mathrm{CO}_{2}$ no interior das instalações é de $3.000 \mathrm{ppm}$, segundo NOLL et al. (2003), e de $2.500 \mathrm{ppm}$, conforme FRAME et al. (1999). MIRAGLIOTTA (2005), ao tomar medidas aleatórias durante alguns dias em lotes diversos de frangos de corte, obteve concentrações médias de $\mathrm{CO}_{2}$ iguais ou inferiores a $1.000 \mathrm{ppm}$ no ambiente de alojamento das aves. A concentração habitual de $\mathrm{CO}_{2}$ na atmosfera encontra-se em 200 a $750 \mathrm{ppm}$. A autora concluiu da mesma forma para o presente trabalho, que a concentração deste gás não representou situações de risco, tanto para os animais quanto para os trabalhadores, visto que, para ambas as situações, o limite máximo está situado em 3.000 e 5.000 ppm, respectivamente (Tabela 2).

Outros autores constataram que contaminantes aéreos indesejados e suas trajetórias diferem em função do sistema de ventilação utilizado (HINDS, 1999), da localização dos ventiladores e da densidade de alojamento (MAGHIRANG et al., 1994). Além disso, SHAW (1994) encontrou uma relação direta entre a velocidade do ar e a intensidade de turbulência na distribuição de variáveis climáticas no interior de aviários.

Para KLOOSTER et al. (1993), a eficácia da renovação de ar pelo sistema de ventilação deve ser bem estruturada, uma vez que o peru é sensível a altas concentrações de amônia e $\mathrm{CO}_{2}$. Caso contrário, os perus jovens ficam sonolentos, deixando de beber água e comer ração, aumentando os índices de mortalidade e refugagem inicial (BONI et al., 2007). Mas conforme SEEDORF et al. (1998), dependendo do manejo utilizado da cama, as taxas de ventilação usualmente utilizadas podem não ser suficientes para manter uma boa qualidade do ar.

Logo, o levantamento das condições de qualidade do ar em cada um dos sistemas de ventilação adotados pela avicultura brasileira, para cada uma das diferentes regiões e estações climáticas do País, é uma necessidade imperativa e urgente das indústrias de produção animal, disponibilizando, com isto, dados de interesse a um inventário nacional, constituindo parte de banco de dados em concentração de gases nocivos pela referida atividade em atendimento das demandas internacionais do mercado, visando à exportação e ao atendimento das leis de preservação ambiental (MENEGALI et al., 2009).

Quanto à temperatura superficial da cama, conforme ABREU (1994), em estudo comportamental de preferência de permanência com frangos de corte, relata que as aves não permanecem sobre a cama com temperaturas superficiais superiores a $36^{\circ} \mathrm{C}$, magnitude essa não alcançada pelos sistemas aqui avaliados (Tabela 2).

Verifica-se, na Figura 2a, que o consumo de energia elétrica (CEE) nos respectivos tratamentos foi maior nos meses de novembro e dezembro. Esse aumento deve-se ao fato do acréscimo das temperaturas externas e do crescimento das aves, acarretando em maior tempo de funcionamento dos sistemas de climatização, pois uma das formas de manejo utilizada com o objetivo de reduzir a sensação térmica das aves é o aumento da velocidade do ar por meio do uso de ventiladores (FLOOD et al., 1991; LACY \& CZARICK, 1992).

De acordo com a Figura $2 \mathrm{~b}$ e segundo resultado da Análise de Variância, comprovou-se a diferença significativa do consumo médio de energia elétrica entre os tratamentos $(\mathrm{P}=0,007)$, em que o tratamento A1 apresentou menor CEE. Esse resultado cabe ser ressaltado, pois o A1 foi o aviário programado para acionar os sistemas de climatização com temperaturas superiores em $4{ }^{\circ} \mathrm{C}$ a programação do sistema A2. A diferença média de consumo entre esses dois aviários foi de $130,5 \mathrm{kWh}$, o que hoje representaria para a região de condução desse estudo (custo do $\mathrm{kWh}=0,35$ reais), $\mathrm{R} \$ 45,68$ em economia mensal. POGI \& PIEDADE JÚNIOR (1991) justificam, inclusive, que uma mínima redução no CEE deve ser valorizada, pois na atividade avícola a energia elétrica é imprescindível e cada vez mais se torna necessária sua racionalização frente aos custos que vem alcançando. 

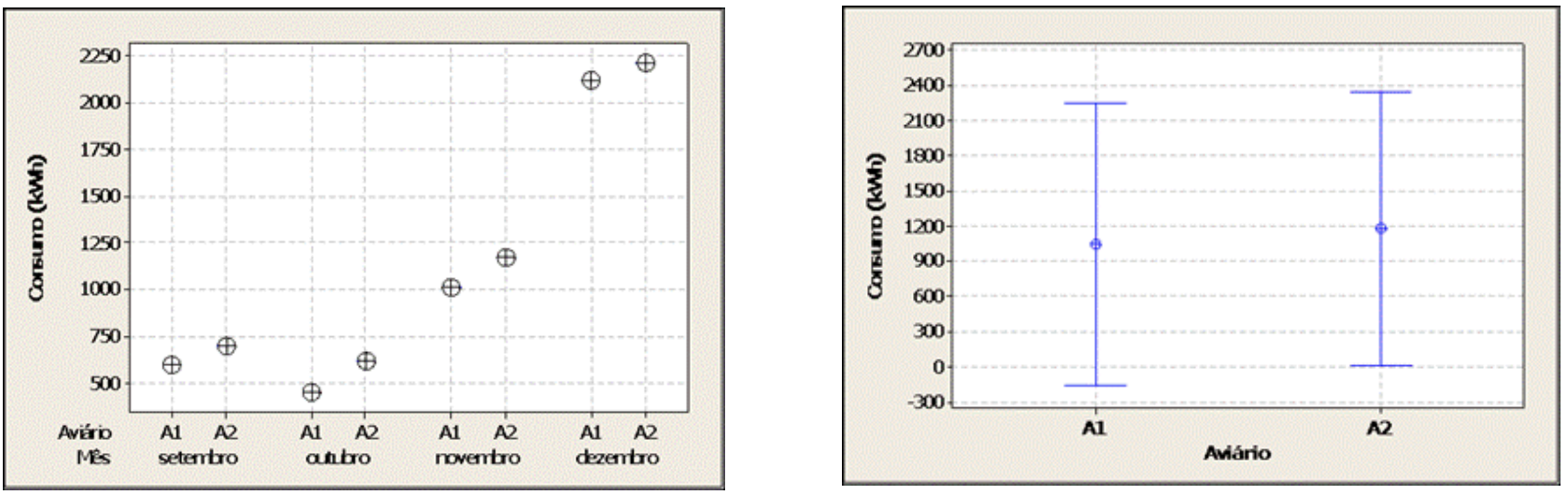

FIGURA 2. Consumo de energia elétrica (kWh) ao longo dos meses (a) e CEE médio (b) por aviário. Electric power consumption $(\mathrm{kWh})$ over the months (a) and average $\mathrm{CEE}$ (b) per poultry houses.

De acordo com resultados obtidos por HUGHES \& WU (1985), a operação adequada da potência dos ventiladores pode produzir uma economia de energia elétrica na ordem de 8 a $12 \%$ e para FLOOD et al. (1998), a operação de ventiladores em baixas velocidades pode efetivamente eliminar gradientes verticais de temperatura.

Conforme SIMMONS \& LOTT (1992), ao desenvolverem um sistema de acionamento dos ventiladores por meio de anemômetros, visando a aproveitar a ventilação natural, relatam que se pode reduzir o tempo de funcionamento dos ventiladores, em média, em $50 \%$, obtendo uma economia de energia elétrica em semelhante proporção. Além disso, constataram que a redução no tempo de funcionamento dos equipamentos pode atingir uma economia de energia na ordem de cinco vezes, ou seja, mais de $160 \mathrm{kWh}$ por dia, por aviário, semelhante ao valor obtido neste estudo (da ordem $130 \mathrm{kWh}$ ).

TURCO et al. (1998), em estudo com frangos de corte, relataram que o consumo de energia elétrica varia em função dos equipamentos utilizados (motor, tamanho e material das pás, etc.) e da programação de acionamento adotada. Além disso, verificaram com seus resultados que, racionalizando o dispêndio de energia elétrica, por meio da escolha de melhores conjuntos motorventilador nos aviários, pode-se alcançar uma economia mensal no custo operacional de eletricidade de R\$ 89,79/aviário. BUENO \& ROSSI (2006), comparando dois sistemas de ventilação para frangos de corte, constataram que os índices de eficiência energética foram baixos em ambos os galpões, o que indica que se faz necessária a implementação, nesses sistemas avícolas, de técnicas de utilização racional e de conservação de energia elétrica.

De acordo com CAILLE (1982), o CEE do conjunto motor-ventilador depende do motor utilizado e das horas de uso. Esse mesmo autor verificou que um motor de $1 / 8 \mathrm{cv}$ consome $0,186 \mathrm{kWh}$. No entanto, para SCHMIDT (1976), um sistema de ventilação ambiente para frangos de corte consome de 0,2 a 0,4 kWh. Percebe-se, aqui, a problemática do CEE na produção de perus, em que as aves, quando adultas, necessitam de maior resfriamento ambiental em comparação com frangos de corte.

Quanto aos dados produtivos, na Tabela 3, apresentam-se os resultados obtidos de peso vivo final, ganho de peso semanal, conversão alimentar e mortalidade. Percebe-se que o tratamento A1 apresentou melhor conversão alimentar, porém maior mortalidade em comparação ao A2.

Conforme o resultado da Análise de Variância, comprova-se que não houve diferença significativa, entre os tratamentos, para o GP semanal $(\mathrm{P}=0,994)$ e o peso médio $(\mathrm{P}=0,987)$. Porém, segundo o teste Qui-quadrado, houve diferença significativa entre os tratamentos para os percentuais de mortalidade $(\mathrm{P}=0,000)$ dos lotes alojados (Tabela 4), em que o tratamento A1 apresentou os maiores percentuais. 
TABELA 3. Resultados produtivos obtidos nos dois tratamentos. Productive results obtained in both treatments.

\begin{tabular}{ccccccc}
\hline Aviário & $\begin{array}{c}\text { Peso } \\
\text { Médio }(\mathrm{kg})\end{array}$ & $\begin{array}{c}\text { Ganho de Peso } \\
\text { Semanal }(\mathrm{kg})\end{array}$ & $\begin{array}{c}\text { Aves } \\
\text { Abatidas }\end{array}$ & $\begin{array}{c}\text { Consumo de } \\
\text { Ração }(\mathrm{kg})\end{array}$ & $\begin{array}{c}\text { Conversão } \\
\text { Alimentar } \\
\left(\mathrm{kg} \mathrm{kg}^{-1}\right)\end{array}$ & $\begin{array}{c}\text { Mortalidade } \\
(\%)\end{array}$ \\
\hline A1 & 15,061 & 0,239 & 4.244 & 164.650 & 2,576 & 5,6889 \\
A2 & 15,755 & 0,254 & 4.344 & 180.560 & 2,638 & 3,4667 \\
\hline
\end{tabular}

TABELA 4. Teste Qui-quadrado para o percentual de mortalidade frente aos tratamentos. Chisquare test for the percentage of mortality compared to the treatments.

\begin{tabular}{cccc}
\hline Aviário & Abatidas & Mortalidade & Total \\
\hline & 4.244 & 256 & 4.500 \\
1 & 4.294 & 206,0 & \\
& 0,582 & 12,136 & 4.500 \\
2 & 4.344 & 156 & \\
\hline Total & 4.294 & 206,0 & 9.000 \\
\hline
\end{tabular}

Chi-Sq $=25,436 ;$ GL $=1 ;$ P-Valor $=0,000$

Quanto às condenações, na Tabela 5, apresentam-se os percentuais de incidências de aerossaculite, dermatose e artrite, frente aos distintos tratamentos. Observa-se que o A1 apresentou o maior percentual de incidência de aerossaculite, porém os menores percentuais de dermatose e artrite. Segundo o teste Qui-quadrado, não houve diferença significativa entre os percentuais da incidência de aerossaculite $(\mathrm{P}=0,286)$ e de dermatose $(\mathrm{P}=0,203)$ nas aves alojadas, porém houve diferença significativa para condenações por artrite $(\mathrm{P}=0,000)$.

TABELA 5. Percentual de condenações parciais. Percentage of partial convictions.

\begin{tabular}{ccccc}
\hline Aviário & Aerosaculite $(\%)$ & Dermatose $(\%)$ & Artrite $(\%)$ & Quantidade Condenações Parciais (\%) \\
\hline A1 & 5,702 & 2,097 & 0,495 & 8,294 \\
A2 & 5,180 & 2,509 & 1,773 & 9,462 \\
\hline
\end{tabular}

Conforme JULIAN (2005), uma das grandes causas da incidência de aerossaculite em lotes de aves comerciais é a baixa qualidade do ar, denotada por altas concentrações de $\mathrm{NH}_{3}, \mathrm{CO}_{2}$ e outros gases. As análises anteriores sobre as variáveis climáticas comprovam que não houve diferença nas concentrações de $\mathrm{CO}_{2}$ entre os tratamentos, assim como para a variável incidência de aerossaculite, corroborando o autor.

Quanto à incidência de dermatose, esses resultados estão em consonância com BRUCE et al. (1990), que observaram correlação positiva entre essa enfermidade com o percentual de UR do ar. Neste estudo, tanto as médias de UR do ar como a incidência de dermatose não apresentaram diferenças significativas entre os tratamentos. Além disso, EKSTRAND \& ALGERS (1997) observaram que a incidência de dermatose em perus foi menor no tratamento em que o avicultor acrescentava mais cama durante o crescimento do lote, manejo não adotado neste estudo.

Conforme DICKEL et al. (2006), artrite é o aumento de volume e supuração nas articulações. Várias são as causas que podem promover o aparecimento de artrites, sendo que as principais são de origem nutricional ou infecciosa, que podem ser produzidas por vírus e bactérias. A causa de o A2 apresentar maior incidência desta enfermidade pode estar atrelada às menores temperaturas ambientais (consequentemente maior UR do ar). Porém, FRAME et al. (1999) afirmam que problemas locomotores e aerossaculite causados por cama úmida, ambiente úmido e baixa 
qualidade do ar são muito mais danosos economicamente que uma conta um pouco maior de energia elétrica.

Com o exposto neste estudo, percebe-se que o sistema de climatização causa efeitos em distintos setores da produção animal: sanidade, produtivo e ambiental, corroborando recentes trabalhos que igualmente comprovaram esses efeitos na produção avícola (SANTOS et al., 2005) e em demais espécies: fêmeas suínas e leitões na maternidade (TOLLON \& NÄ̈̈S, 2005) e bovinos de leite (MARTELO et al., 2004).

\section{CONCLUSÕES}

Ambos os sistemas de acionamento da climatização não atingiram as temperaturas desejadas para a criação de perus em terminação, porém atingiram concentrações adequadas de dióxido de carbono e mantiveram a umidade relativa dentro dos valores recomendados pela literatura.

O sistema de acionamento programado para ser acionado mais tardiamente consumiu menos energia elétrica, atingiu melhor conversão alimentar das aves, menor incidência de artrite, porém maior percentual de mortalidade, apresentando melhor custo-benefício para a fase final de criação de perus, em épocas de baixa umidade relativa do ar.

\section{REFERÊNCIAS}

ABREU, P.G. Sistemas de aquecimento em piso, com resistência elétrica, para criação de aves. 1994. 108 f. Dissertação (Mestrado em Construções Rurais) - Faculdade de Engenharia Agrícola, Universidade Federal de Viçosa, Viçosa, 1994.

ANDONOV, K.; DASKALOV, P.; MARTEV, K. A new approach to controlled natural ventilation of livestock buildings. Biosystems Engineering, London, v.84, n.1, p.91-100, 2003.

ASHRAE. AMERICAN SOCIETY OF HEATING, REFRIGERATING, AND AIR CONDITIONING ENGINEERS. Handbook of fundamentals. Georgia: ASAE, 2002. 180 p.

BONI, I.J.; KONZEN, F.A.; VIZZOTTO, M.A. Manejo reprodutivo de perus. Revista Brasileira de Reprodução Animal, Belo Horizonte, v.31, n.3, p.328-332, 2007.

BRUCE, D.W.; MCILROY, S.G.; GOODALL, E.A. Epidemiology of a contact dermatitis of broilers. Avian Pathology, Huntingdon, v.19, p.532-537, 1990.

BUENO, L.; ROSSI, L.A. Comparação entre tecnologias de climatização para criação de frangos quanto à energia, ambiência e produtividade. Revista Brasileira de Engenharia Agrícola e Ambiental, Campina Grande, v.10, n.2, p.497-504, 2006.

CAILLE, R.E. Aparelhos elétricos. São Paulo: CESP, 1982. 64 p.

CARVALHO, T.M. Variabilidade espacial de propriedades físico-hídricas de um Latossolo Vermelho-Amarelo através da geoestatística. 1991. 178 f. Tese (Doutorado em Ciência do Solo) Universidade de Lavras, Lavras, 1991.

CAVALCHINI, L.G. El Pavo. Madrid: Mundi-Prensa, 1985. 308 p.

DICKEL, L.; DARMAILLACQ, A.; CHICHERY, R.; SHASHAR, N. Early familiarization overrides innate prey preference in newly hatched Sepia officinalis cuttlefish. Animal Behaviour, v.71, n.3, p.511-514, 2006.

EKSTRAND, C.; ALGERS, B. Rearing conditions and foot-pad dermatitis in Swedish turkey poults. Acta Veterinaria Scandinavica, Copenhagen, v.38, p.167-174, 1997.

FLOOD, C.A.; TRUMBULL, R.D.; KOON, J.L.; BREWER, R.N. Partitioned ventilation control for broilers. Transactions of the ASAE, St. Joseph, v.34, n.6, p.2.541-2.549, 1991. 
FLOOD, C.A.J.; KOON, J.L.; TRUMBULL, R.D.; BREWER, R.N. Energy savings with ceiling fans in broiler houses. Applied Engineering in Agriculture, St. Joseph, v.14, n.3, p.305-309, 1998.

FRAME, D.D.; BUCKNER, R.E.; ANDERSON, G.L. Causes and control of spontaneous cardiomyopathy or roundheart disease in Utah turkeys. Logan: Utah State University, 1999. 9 p. (Bulletin AG506).

HINDS, W.C. Aerosol technology. New York: John Wiley \& Sons, 1999. 120 p.

HUGHES, H.A.; WU, J., JINKANG, W. Ceiling fans for broiler houses. St. Joseph: ASAE, 1985. (Paper, 85).

JULIAN, R.J. Production and growth related disorders and other metabolic diseases of poultry - A review. The Veterinary Journal, v.169, p.350-369, 2005.

KLOOSTER, C.E.; VAN'T, P.F.; ROELOFS, M.M.; GIJSENET, P.A.M. Positioning air inlet and air outlet to reduce dust exposure in pig buildings. In: INTERNATIONAL SYMPOSIUM ON LIVESTOCK ENVIRONMENT, 4., 1993, St. Joseph. Proceedings... St. Joseph: ASAE, 1993. p.754-759.

LACY, M.P.; CZARICK, M. Tunnel-ventilated broiler houses: broiler performance and operating costs. Journal applied Poultry Research, Athens, v.1, p.104-109, 1992.

LEE, I.; BYOENG-KI, Y.; KYU-HONG, C.; JONG-GIL, J.; GYEONG-WON, K. Study of internal climate of naturally and mechanically ventilated broiler houses. In: ASAE ANNUAL

INTERNATIONAL MEETING, 2003, Las Vegas. Proceedings... Las Vegas: ASAE, 2003. 1 CDROM.

MAGHIRANG, R.G.; MANBECK, H.B.; PURI, V.M. Numerical simulation of particle transport in slot-inlet ventilated airspaces. Transactions of the ASAE, St. Joseph, v.37, n.5, p.1.607-1.612, 1994.

MANUAL DE BOAS PRÁTICAS DA NICHOLAS. Disponível em: <http://nicholas-turkey.com>. Acesso em: 10 dez. 2007.

MARTELLO, L.S.; JÚNIOR, H.S.; PINHEIRO, M.G.; SILVA, S.L.; JÚNIOR, L.C.R. Avaliação do microclima de instalações para gado de leite com diferentes recursos de climatização.

Engenharia Agrícola, Jaboticabal, v.24, n.2, p.263-273, 2004.

MENEGALI, I.; TINÔCO, I.F.F.; BAÊTA, F.C.; CECON, P.R.; GUIMARÃES, M.C.C.;

CORDEIRO, M.B. Ambiente térmico e concentração de gases em instalações para frangos de corte no período de aquecimento. Revista Brasileira de Engenharia Agrícola e Ambiental, Campina Grande, v.13, p.984-990, 2009. Suplemento.

MIRAGLIOTTA, M.Y. Avaliação das condições do ambiente interno em dois galpões de produção comercial de frangos de corte, com ventilação e densidade populacional diferenciados. $2005.258 \mathrm{f}$. Tese (Doutorado em Engenharia Agrícola) - Faculdade de Engenharia Agrícola, Universidade Estadual de Campinas, Campinas, 2005.

MUTAF, S.; ALKAN, S.; SEBER, N. The effects of natural ventilation air exchange on psychrometric results in poultry houses in hot environment and design characteristics. CIGR Journal of Scientific Research and Development. Manuscript BC 03 023, 2004. (Agricultural Engineering International: The CIGR EJournal).

NOLL, S.L.; NAGARAJA, K.V.; HALVORSON, D.A.; JANNI, K.A. Air quality in Turkey production. Minneapolis: University of Minnesota, 2003. 8 p. Extension Service.

POGI, R.C.; PIEDADE, J.R.C. Energia elétrica em atividades ligadas à avicultura. Energia na Agricultura, Botucatu, v.6, p.28-34, 1991. 
SANTOS, P.A.; JUNIOR, T.Y.; TEIXEIRA, V.H.; FERREIRA, L. Ambiente térmico no interior de modelos de galpões avícolas em escala reduzida com ventilação natural e artificial dos telhados. Engenharia Agrícola, Jaboticabal, v.25, n.3, p.575-584, 2005.

SCHMIDT, W. Guia de instalações elétricas rurais. São Paulo: SIEMENS, 1976. v.8. 80 p.

SEEDORF, J.; HARTUNG, J.; SCHRÖDER, M.; LINKERT, K.H.; PEDERSEN, S.; TAKAI, H.; JOHNSEN, J.O.; METZ, J.H.M.; GROOT KOERKAMP, P.W.G.; UENK, G. H.; PHILLIPS, V.R.; HOLDEN, M.R.; SNEATH, R.W.; SHORT, J.L.L.; WHITE, R.P.; WATHES, C.M.. A Survey of Ventilation Rates in Livestock Buildings in Northern Europe. Journal of Agricultural Engineering Research, London, v.70, n.1, p.39-47, 1998.

SENTHILSELVAN, A.; DOSMAN, J.A.; KIRYCHUK, S.P.; BARBER, E.M.; RHODES, C.S.; ZHANG, Y.; HURST, T.S. Accelerated lung function decline in confinement swine building workers. Chest, Chicago, v.111, n.6, p.1.733-1.741, 1997.

SEO, H.I.; LEE, I.B.; CHANG, P.W.; HWANG, H.S.; HONG, S.W.; LEE, S.Y. Study on ventilation system of naturally ventilated broiler house by aerodynamic approach. In: ASABE MEETING PRESENTATION, 2006, Oregon. Proceedings... Paper 06401.

SHAW, B.W. Use of a convective emission chamber to study particle resuspension. 1994. $145 \mathrm{f}$. Thesis (Ph.D.) - University of Illinois, Urbana, 1994.

SIMMONS, J.D.; LOTT, B.D. Wind-activated fan shutoff to reduce electricity required for summer ventilation. Applied Engineering in Agriculture, St. Joseph, v.8, n.6, p.851-854, 1992.

TOLON,Y.B.; NÄÄS, I.A. Avaliação de tipos de ventilação em maternidade de suínos. Engenharia Agrícola, Jaboticabal, v.25, n.3, p.565-574, 2005.

TURCO, J.E.P.; MILANI, A.P.; FURLAN, R.L.; MACARI, M. Análise do consumo de energia elétrica e eficiência de conjuntos motor-ventilador utilizados na avicultura brasileira. Engenharia Agrícola, Jaboticabal, v.18, n.13-19, 1998.

UBA. UNIÃO BRASILEIRA DE AVICULTURA. Relatório anual 2009. Disponível em: <http://www.uba.org.br/ubanews_files/relatorio_uba_06_07_baixa_1.pdf>.

VAN DER HEL, W.; VERSTERGEN, M.W.A.; PIJLS, L.; VAN KAMPEN, M. Effect of two day temperature exposure of neonatal broiler chicks on growth performance and body composition during two weeks at normal conditions. Poultry Science, Champaign, v.71, p. 2.014-2.021, 1992.

WANG, X.; ZHANG, Y. Development of a critical air flow venturi for air sampling. Journal of Agricultural Engineering Research, London, v.73, p.257-264, 1999.

WANG, X.; ZHANG, Y.; RISKOWSKI, G.L. Dust spatial distribution in a typical swine building. In: INTERNATIONAL SYMPOSIUM ON "DUST CONTROL FOR ANIMAL PRODUCTION FACILITIES, 1999, Aarhus, Denmark. Proceedings... Denmark: Danish Institute of Agricultural Sciences.

WATHES, C.M.; PHILLIPS, V.R.; HOLDEN, M.R.; SNEATH, R.W.; SHORT, J.L.; WHITE, R.P.; HARTUNG, J.; SEEDORF, J.; SCHRODER, M.; LINKERT, K.D.; PEDERSEN, S.; TAKAI, H.; JOHNSEN, J.O.; GROOT KOERKAMP, P.W.G.; UENK, G.H.; METZ, J.H.M.; HINZ, T.; CASPARY, V.; LINKE, S. Emission of aerial pollutants in livestock buildings in Northern Europe: overview of a multinational project. Journal of Agricultural Engineering Research, Silsoe, v.70, n.1, p.3-9, 1998.

XIN, H. CHEPETE, H.G.; SHAO, J.; SELL, J.L. Heat and moisture production and minimum ventilation requirements of tom turkeys during brooding-growing period. Transactions of the ASAE, St. Joseph, v.41, n.5, p.1.489-1.498, 1998. 
YALÇIN, S.; TESTIK, A.; OZKAN, S.; SETTAR, P.; CELEN, F.; CAHANER, C. Performance of naked neck and normal broilers in hot, warm, and temperate climates. Poultry Science, Champaign, v.76, n.7, p.930-937, 1997.

ZEDJA, J.E.; BARBER, E.; DOSMAN, J.A.; OLENCHOCK, S.A.; MCDUFFIE, H.H.; RHODES, C.; HURST, T. Respiratory health status in swine producers relates to endotoxin exposure in the presence of low dust levels. Journal of Occupational and Environment Medicine, v. 36, n.1, p.4956,1994 .

ZHANG, Y.; WANG, X.; RISKOWSKI, G.L.; CHRISTIANSON, L.L. Quantifying ventilation effectiveness for air quality control. Transactions of the ASAE, St. Joseph, v.44, n.2, p.385-390, 2001. 